\title{
Produção de cultivares de morango, utilizando túnel baixo em Pelotas
}

\author{
Roberto Pedroso de Oliveira* ${ }^{\text {, }}$ Walkyria Bueno Scivittaro ${ }^{1}$, Paulo Sérgio Gomes da Rocha ${ }^{2}$
}

\section{RESUMO}

O objetivo deste trabalho foi avaliar o desempenho produtivo de novos cultivares de dia-curto de morangueiro ('Earlibrite', 'Sabrosa' e 'Florida Festival'), nas condições edafo-climáticas de Pelotas (RS), tendo como padrão o cultivar 'Camarosa', usando-se túnel baixo. O experimento foi realizado com sistema de irrigação por gotejamento. O transplantio das mudas foi realizado em maio de 2008, sendo utilizadas mudas procedentes da região da Patagônia, na Argentina. O delineamento experimental foi de blocos casualizados, com parcelas subdivididas no tempo, com quatro repetições. As unidades experimentais foram constituídas por 42 plantas, que foram avaliadas, semanalmente, quanto ao número, massa da matéria fresca e produção de frutos, no período de agosto a dezembro de 2008. Nas avaliações, consideraram-se apenas os frutos comercializáveis, descartando-se aqueles com defeitos e com massa inferior a $5 \mathrm{~g}$. Verificou-se que a produção acumulada de frutos por planta do 'Sabrosa' é maior que a do 'Florida Festival' e do 'Earlibrite', menor, porém, que a do 'Camarosa'. O número de frutos produzidos por planta do 'Sabrosa' e do 'Florida Festival' é maior que o do 'Earlibrite', menor, porém, que o do 'Camarosa'. Quanto à produção de massa da matéria fresca média dos frutos, o 'Sabrosa' e o 'Camarosa' são superiores a do 'Florida Festival' e a do 'Earlibrite'. Apesar de apresentarem padrões de desempenho produtivo diferenciados, os três novos cultivares de morangueiro testados mostraram-se com alto potencial produtivo, constituindo-se em interessantes alternativas de produção para os agricultores de Pelotas.

Palavras-chave: Fragaria x ananassa Duch., dia-curto, produtividade, período de colheita.

\section{ABSTRACT}

\section{Yield of strawberry cultivars under a low tunnel system in Pelotas, RS, Brazil}

The objective of this study was to evaluate the yield of new short-day strawberry cultivars (Earlibrite, Sabrosa and Florida Festival) and to compare them with cv. 'Camarosa' under soil and climatic conditions of Pelotas (RS) using a low tunnel system using drip irrigation. The strawberry plants originated from the Patagonia Region, Argentina, were transplanted in May 2008. The experiment was arranged in a complete randomized block design in split plots, with four replications. The experimental unit consisted of 42 plants. The number of fruits, yield and fresh fruit mass were recorded weekly from August to December 2008, considering only the fruits that weighed above $5 \mathrm{~g}$ without defects. The cultivar 'Sabrosa' provided higher yield than 'Florida Festival' and 'Earlibrite', but lower than 'Camarosa'. 'Sabrosa' and 'Florida Festival' produced larger number of fruits per plant than 'Earlibrite', but smaller than Camarosa. 'Sabrosa' and 'Camarosa' produced higher average fruit fresh mass than 'Florida Festival' and 'Earlibrite'. Although showing different yield performances, the three new cultivars showed high yield potential and, therefore, they can be indicated as new alternatives for the strawberry farmers of Pelotas.

Key words: Fragaria x ananassa Duch., harvest period, short-day, yield.

Recebido para publicação em 04/10/2010 e aprovado em 19/09/2011

'Engenheiro-Agrônomo, Doutor. Embrapa Clima Temperado, C. Postal 403, 96001-970 Pelotas, RS, Brasil. roberto.pedroso@cpact.embrapa.br, walkyria.scivittaro@cpact.embrapa.br. *Autor correspondente

22Engenheiro-Agrônomo, Doutor. Bolsista Pós-doutorado CNPq. p.sergio.r@uol.com.br 


\section{INTRODUÇÃO}

O morangueiro (Fragaria x ananassa Duch) é produzido e apreciado nas mais variadas regiões do mundo, sendo a espécie do grupo das pequenas frutas de maior expressão econômica (Oliveira et al., 2005). A planta pertence à família das Rosaceas, sendo um híbrido resultante das espécies americanas $F$. chiloensis, $F$. virginiana e $F$. ovalis e da europeia $F$. vesca (Ronque, 1998). No Brasil, a cultura está difundida em regiões de clima temperado e subtropical, onde são produzidos frutos para consumo in natura e industrialização (Santos, 2003).

A produção do morangueiro é influenciada pela interação dos fatores temperatura e fotoperíodo, sendo os cultivares de dia-curto aqueles em que a indução floral ocorre no final de verão ou início do outono, quando os dias são mais curtos $(<14$ horas) e as temperaturas mais amenas $\left(<16^{\circ} \mathrm{C}\right)$ (Santos, 2003). Os cultivares de morangueiro de dia-curto mais utilizados na região sul do Brasil são o 'Camarosa' e o 'Camino Real' (Oliveira et al., 2008), enquanto, no restante do país, o 'Oso Grande' é o de maior expressão econômica (Antunes \& Reisser Júnior, 2007).

Em razão da diversidade edafo-climática existente no país, o pequeno número de cultivares disponível tem sido um dos principais obstáculos ao desenvolvimento da cultura do morangueiro. Assim, adquire grande importância o incentivo aos programas nacionais de melhoramento genético e de introdução e avaliação de cultivares gerados em outros países.

O ‘Camarosa', obtido na Universidade da Califórnia, foi registrado no Brasil em 1999. Suas plantas são vigorosas e as folhas grandes e de coloração verde-escura; de ciclo precoce, as plantas apresentam alta capacidade de produção de frutas, que são grandes, uniformes, de coloração vermelho-escura, polpa firme e sabor subácido, sendo indicado, tanto para consumo in natura, quanto para a industrialização (Santos, 2003; Shasta Nursery, 2011).

Em 2007, começaram a chegar ao Brasil as primeiras mudas dos cultivares de dia-curto Earlybrite e Florida Festival, provenientes da Argentina. O 'Earlibrite' resulta do cruzamento entre 'Rosa Linda' e 'FL 90-38', tendo sido obtido na Universidade da Flórida, em 1993, muito embora a liberação para plantio nos Estados Unidos só tenha ocorrido em 2000. As plantas desse cultivar são bem compactas, a produção é bastante precoce e os frutos apresentam tamanho grande ( $>20 \mathrm{~g}$ ), formato globoso-cônico, coloração vermelho-alaranjada, textura moderadamente firme e sabor agradável, sendo de fácil colheita (Chandler et al., 2000a). O 'Florida Festival' também foi obtido na Universidade da Flórida, em 1995, sendo resultante do cruzamento entre 'Rosa Linda' e 'Oso Grande'. A liberação para o plantio nos Estados Unidos ocorreu em 2000.
As plantas desse cultivar são vigorosas e produtivas; os frutos apresentam formato cônico, tamanho médio $(<20$ g), coloração vermelha uniforme, textura firme e excelente sabor (Chandler et al., 2000b). Assim como o 'Earlibrite', o 'Florida Festival' é susceptível à antracnose (Colletotrichum sp.) e apresenta relativa tolerância ao mofo-cinzento (Botrytis cinerea) e ao oídio (Sphaerotheca macularis f. sp. fragariae) (Chandler et al., 2000a, b).

O cultivar Sabrosa, também conhecido por Candonga, foi obtido em Cartaya, Huelva, na Espanha, pela empresa PLANASA (Plantas de Navarra S.A.), sendo originário do cruzamento entre as seleções 92-38 e 86-032 (Planasa, 2011). Este cultivar tem sido recomendado na Europa desde 2004. As plantas são vigorosas, compactas e de hábito de crescimento ereto, sendo o sistema radicular resistente a patógenos; os frutos são muito atrativos, de formato cônico, coloração vermelha-brilhante, polpa firme e sabor agradável com grande quantidade de açúcares, sendo pouco deformados em função da alta fertilidade do pólen. Apresenta alta resistência ao mofo- cinzento e, especialmente, a Oidium fragariae e a Phytophthora fragariae f. sp. fragariae (Inotalis, 2011).

Os desempenhos produtivos satisfatórios obtidos com os cultivares Earlibrite e Florida Festival, nos Estados Unidos (Chandler et al., 2000a, b), e com o cv. Sabrosa, na Espanha (Inotalis, 2011; Planasa, 2011), denotam a importância da avaliação desses materiais no Brasil.

O objetivo deste trabalho foi avaliar a adaptabilidade dos cultivares de morangueiro Earlibrite, Sabrosa e Florida Festival às condições edafo-climáticas de Pelotas-RS, sob condições de produção em túnel baixo, utilizando-se, como padrão de referência, o cultivar Camarosa.

\section{MATERIAL E MÉTODOS}

O experimento foi realizado no município de Pelotas, RS, localizado a $31^{\circ} 46^{\prime} 19^{\prime \prime} \mathrm{S}, 52^{\circ} 20^{\prime} 33^{\prime \prime} \mathrm{O}, 245 \mathrm{~m}$ de altitude e temperatura média anual de $17,6^{\circ} \mathrm{C}$. Foram avaliados quatro cultivares de morangueiro de dia-curto ('Camarosa', 'Earlibrite', 'Sabrosa' e 'Florida Festival'), sendo o primeiro considerado como padrão de referência para a região. Utilizaram-se mudas procedentes da região da Patagônia, na Argentina.

O transplantio das mudas foi realizado em 02 de maio de 2008, para canteiros de $1,2 \mathrm{~m}$ de largura por $0,15 \mathrm{~m}$ de altura, espaçados entre si em $0,8 \mathrm{~m}$. O solo da área experimental, um Argissolo Vermelho eutrófico típico (Santos et al., 2006), foi previamente corrigido, quanto à acidez, para $\mathrm{pH}_{\text {(agua) }} 6,0$ (Sociedade, 2004). O espaçamento entre linhas e entre plantas foi de $0,35 \mathrm{~m}$, sendo dispostas três linhas por canteiro. Os canteiros foram revestidos com filme de polietileno preto, com $30 \mu \mathrm{m}$ de espessura. Utilizou-se o 
sistema de produção em túneis plásticos, com $0,5 \mathrm{~m}$ de altura na parte central.

As plantas de morangueiro foram irrigadas por gotejamento, de acordo com a necessidade hídrica, durante todo o período de cultivo. O controle das plantas daninhas e a remoção de folhas secas ou com sintomas de doenças, de estolões e de frutos danificados ou com defeitos, foram realizados manualmente. Semanalmente, as plantas foram fertirrigadas, com aplicações alternadas dos fertilizantes $\mathrm{KSC}^{\circledR}{ }^{\circledR}\left(14 \% \mathrm{~N}, 40 \% \mathrm{P}_{2} \mathrm{O}_{5}, 5 \% \mathrm{~K}_{2} \mathrm{O}\right.$, $13 \% \mathrm{SO}_{3}, 0,1 \% \mathrm{~B}, 0,05 \% \mathrm{Cu}, 0,1 \% \mathrm{Fe}, 0,01 \%$ Mo e $0,1 \%$ $\mathrm{Zn}), \mathrm{KSC}^{\circledR}\left(8 \% \mathrm{~N}, 16 \% \mathrm{P}_{2} \mathrm{O}_{5}, 42 \% \mathrm{~K}_{2} \mathrm{O}, 0,1 \% \mathrm{~B}, 0,1 \% \mathrm{Fe}\right.$, $0,05 \% \mathrm{Mn}$ e $0,1 \% \mathrm{Zn}$ ) e nitrato de cálcio (500 g por 1000 plantas). O tratamento fitossanitário incluiu três aplicações com iprodione e duas com azoxystrobin, para o controle de doenças fúngicas. Também, foi realizada uma aplicação com abamectina, para o controle de ácaros, e foram utilizadas iscas para o controle de broca-dos-frutos (Lobiopa insularis) e de camundongos (Oliveira et al., 2008).

Durante o período de produção dos frutos, a umidade relativa do ar média e as médias das temperaturas mínimas e máximas mensais foram, respectivamente, de $74 \%, 8,2^{\circ} \mathrm{C}$ e $18,0^{\circ} \mathrm{C}$, em agosto; $72 \%, 9,9^{\circ} \mathrm{C}$ e $18,8^{\circ} \mathrm{C}$, em setembro; $70 \%, 13,1^{\circ} \mathrm{Ce} 22,8^{\circ} \mathrm{C}$, em outubro; $70 \%, 17,2^{\circ} \mathrm{C}$ e $27,0^{\circ} \mathrm{C}$, em novembro, e $66 \%, 17,1^{\circ} \mathrm{C}$ e $27,5^{\circ} \mathrm{C}$, em dezembro. Estas informações, assim como os dados climatológicos diários completos referentes ao período completo de cultivo, encontram-se disponíveis na Estação Agroclimatológica de Pelotas (2011).

Para as avaliações, os tratamentos foram distribuídos em delineamento experimental de blocos casualizados, com parcelas subdivididas no tempo e quatro repetições, sendo as unidades experimentais constituídas por 42 plantas. O período de colheita estendeu-se de agosto a dezembro de 2008, sendo realizada uma avaliação por semana, totalizando 20 avaliações. Em cada uma, foram determinados a produção e o número de frutos por unidade experimental. A massa de matéria fresca média dos frutos foi calculada pela razão entre essas duas variáveis. Foram determinados, também, a produção acumulada, o número total e a massa média de frutos, considerando-se o período integral de colheita. Em ambas as avaliações, consideraram-se apenas os frutos comercializáveis, descartando-se aqueles com defeitos e com massa inferior a $5 \mathrm{~g}$ (Oliveira \& Scivittaro, 2008). Para a análise estatística, agruparam-se os dados a cada quatro semanas, perfazendo cinco períodos de avaliação, correspondendo aos meses de agosto a dezembro. Os dados foram submetidos à análise de variância, comparando-se as médias do fator cultivar pelo teste de Tukey $(p<0,05)$ e, do fator período de avaliação, por análise de regressão polinomial.

\section{RESULTADOS E DISCUSSÃO}

Os primeiros frutos dos cultivares estudados começaram a ser colhidos na primeira semana do mês de agosto de 2008, 90 dias após o transplantio das mudas, correspondendo ao final do inverno (umidade relativa média mensal do ar de $74 \%, 8,2^{\circ} \mathrm{C}$ de temperatura média mínima e $18,0^{\circ} \mathrm{C}$ de temperatura média máxima). Este resultado assemelha-se àquele descrito por Singh et al. (2008), que verificaram o início da produção de 25 cultivares de morangueiro, incluindo o 'Camarosa', do $91^{\circ}$ ao $94^{\circ}$ dia após o transplantio das mudas, em condições subtropicais, na Índia.

Ao longo das 20 semanas de colheita, o 'Camarosa' apresentou maior produção acumulada de frutos comercializáveis (1156,3 g / planta), em relação aos demais cultivares estudados (Tabela 1). Estes valores foram semelhantes aos obtidos por Oliveira et al. (2008), na safra de 2007, nas mesmas condições de cultivo, demonstrando tratar-se de um cultivar muito produtivo no extremo sul do Brasil. O cultivar Sabrosa foi o segundo em produção acumulada (925,6 g / planta), seguido pelo 'Florida Festival' (774,0 g / planta) e pelo 'Earlibrite' (606,8 g / planta) (Tabela 1). O maior potencial produtivo do 'Camarosa' em relação ao 'Earlibrite' e ao 'Florida Festival' já havia sido descrito, na Flórida, por Chandler et al. (2000a, b). Estes resultados demonstram a adaptabilidade de cultivares, desenvolvidos em outras regiões e países, ao cultivo no extremo sul do Brasil, já que o 'Sabrosa' provém da Espanha, o 'Camarosa', da Califórnia e o 'Earlibrite' e o 'Florida Festival', da Flórida. As produtividades obtidas neste trabalho para os quatro cultivares estudados foram excelentes, notadamente quando se compara à média do Estado do Rio Grande do Sul (300-400 g / planta) (Pagot \& Hoffmann, 2003) e aos resultados obtidos, na Flórida, por Chandler \& Sumler Jr. (2002), quais sejam: $491 \mathrm{~g}$ / planta para 'Earlibrite' e $503 \mathrm{~g}$ / planta para 'Florida Festival'. Isto indica adequadas condições de cultivo e de manejo utilizadas.

No período de colheita, o número total de frutos produzidos por planta do 'Camarosa' $(41,5)$ foi superior ao dos demais cultivares estudados. Os cultivares 'Sabrosa' $(33,4)$ e 'Florida Festival' $(31,1)$ não diferiram entre si para esta variável e apresentaram melhor desempenho do que o 'Earlibrite' $(23,5)$. Já em relação à massa da matéria fresca média dos frutos, os cultivares Camarosa e Sabrosa apresentaram, respectivamente, 27,9 e 27,7 g / fruto, com desempenho estatisticamente semelhante entre si e maiores que os cultivares Florida Festival (24,9 g / fruto) e Earlibrite (25,9 g / fruto), que também não diferiram entre si (Tabela 1). Nas mesmas localidade e condições de cultivo (túnel baixo), Oliveira et al. (2008) obtiveram maior número de frutos (55,0 unidades) com menor massa da 
matéria fresca média (19,5 g), para o cultivar Camarosa, embora o potencial de produção desse cultivar tenha-se mantido estável, caracterizando-se pela alta produtividade (Shasta Nursery, 2011). Por sua vez, Leis et al. (2002) relataram massa da matéria fresca média de frutos do 'Camarosa' de 23,5 g, aproximando-se do valor obtido neste trabalho. Já em relação aos demais cultivares estudados, o 'Sabrosa' é descrito como tendo potencial para a produção de frutos com grande massa da matéria fresca média (Planasa, 2011) e, o 'Earlibrite', como produtor de frutos maiores do que o 'Florida Festival', embora essa característica varie com as condições de cultivo (Chandler et al., 2000b).

Independentemente do período de avaliação, o 'Camarosa' apresentou maior produção de frutos (Tabela 1). Na tabela 2, verifica-se que, no primeiro período de colheita, correspondente ao mês de agosto, este cultivar apresentou produção superior a do 'Sabrosa'; já os cultivares Florida Festival e Earlibrite apresentaram produções de frutos intermediárias, não diferindo daquelas verificadas para 'Camarosa' e 'Sabrosa'. Estes resultados contrapõem-se à precocidade de produção do 'Earlibrite' e do 'Florida Festival' em relação ao 'Camarosa', referida por Chandler et al. (2000a), na Flórida, o que pode ser atribuído a diferentes sistemas e condições climáticas durante o cultivo, embora a latitude de Gainesville (29³9'54" N) seja próxima a de Pelotas (3146'19" S). Em setembro, a superioridade em produção de frutos do 'Camarosa' manteve-se, sendo seu desempenho equiparado, apenas, ao do 'Florida Festival'. Já no período, compreendido entre a $9^{\mathrm{a}}$ e $12^{\mathrm{a}}$ semanas de colheita, correspondente ao mês de outubro, a produção de frutos do 'Camarosa' destacouse em relação à de todos os demais cultivares. No mês de novembro, o desempenho produtivo dos cultivares distinguiu-se em dois grupos: o primeiro com maior produção de frutos, representado pelo 'Camarosa' e 'Sabrosa', e, o segundo, com produção menor, representado por 'Florida Festival' e 'Earlibrite'. Este comportamento mantevese praticamente constante no mês de dezembro, com exceção da criação de uma classe intermediária de produção de frutos, representada pelo cultivar Sabrosa (Tabela 2).

Para os cultivares de morangueiro Camarosa, Sabrosa e Florida Festival, o efeito do período de colheita sobre a produção de frutos, que é determinado pela temperatura e pelo fotoperíodo, foi ajustado a modelos quadráticos de regressão. Para o 'Earlibrite', entretanto, a produção de frutos foi crescente durante todo o período de avaliação, não se tendo atingido um valor máximo para a variável dentro do período de colheita acompanhado (Figura 1a). Maiores produções de frutos comerciais por planta foram estimadas, respectivamente, na $21^{\mathrm{a}}, 14^{\mathrm{a}}$ e $19^{\mathrm{a}}$ semana, para os cultivares Camarosa (346,1 g), Florida Festival (222,1 g) e Sabrosa (297,0 g). Embora Santos (2003) afirme que a progressiva elevação das temperaturas médias no período de agosto a dezembro, no sul do Brasil, promova a redução na produção de frutos, particularmente para os cultivares de dia-curto de morangueiro, que dependem do fotoperíodo para florescer, no presente estudo, esse

Tabela 1. Produção acumulada, número total e massa da matéria fresca média de frutos de quatro cultivares de dia-curto de morangueiro (Fragaria x ananassa Duch.)

\begin{tabular}{lccc}
\hline Cultivar & $\begin{array}{c}\text { Produção acumulada } \\
\text { g / planta }\end{array}$ & $\begin{array}{c}\text { Número de frutos } \\
\mathbf{N}^{\mathbf{0}} \text { / planta }\end{array}$ & $\begin{array}{c}\text { Massa da matéria fresca média } \\
\text { g / fruto }\end{array}$ \\
\hline Camarosa & $1156,3 \mathrm{a}$ & $41,5 \mathrm{a}$ & $27,9 \mathrm{a}$ \\
Sabrosa & $925,6 \mathrm{~b}$ & $33,4 \mathrm{~b}$ & $27,7 \mathrm{a}$ \\
Florida Festival & $774,0 \mathrm{c}$ & $31,1 \mathrm{~b}$ & $24,9 \mathrm{~b}$ \\
Earlibrite & $606,8 \mathrm{~d}$ & $23,5 \mathrm{c}$ & $25,9 \mathrm{~b}$ \\
\hline $\mathrm{CV}(\%)$ & 4,9 & 4,0 & 1,8 \\
\hline
\end{tabular}

Médias seguidas de mesma letra, nas colunas, não diferem significativamente entre si pelo teste de Tukey a 5\%.

Tabela 2. Produção de frutos de quatro cultivares de dia-curto de morangueiro (Fragaria $\mathrm{x}$ ananassa Duch), em função do período de colheita

\begin{tabular}{lccccc}
\hline \multirow{2}{*}{ Cultivar } & \multicolumn{5}{c}{ Período de colheita } \\
\cline { 2 - 5 } & Agosto & Setembro & Outubro & Novembro & Dezembro \\
\cline { 2 - 5 } & & \multicolumn{5}{c}{$\mathrm{g} / \mathrm{planta}$} \\
Camarosa & $81,2 \mathrm{a}$ & $112,2 \mathrm{a}$ & $295,4 \mathrm{a}$ & $339,7 \mathrm{a}$ & $327,8 \mathrm{a}$ \\
Sabrosa & $26,2 \mathrm{~b}$ & $51,4 \mathrm{c}$ & $222,4 \mathrm{~b}$ & $372,9 \mathrm{a}$ & $252,7 \mathrm{~b}$ \\
Florida Festival & $58,8 \mathrm{ab}$ & $97,8 \mathrm{ab}$ & $212,0 \mathrm{~b}$ & $270,8 \mathrm{~b}$ & $134,6 \mathrm{c}$ \\
Earlibrite & $52,3 \mathrm{ab}$ & $68,4 \mathrm{bc}$ & $84,3 \mathrm{c}$ & $243,8 \mathrm{~b}$ & $158,6 \mathrm{c}$ \\
\hline
\end{tabular}

$\mathrm{CV}$ (cultivar) $=4,9 \% \quad \mathrm{CV}$ (período avaliação) $=10,9 \%$

Médias seguidas de mesma letra, nas colunas, não diferem significativamente entre si pelo teste de Tukey a 5\%.

Rev. Ceres, Viçosa, v. 58, n.5, p. 625-631, set/out, 2011 
resultado começou a se manifestar, apenas, a partir do quinto período de avaliação (correspondente ao mês de dezembro), o que provavelmente se deva às temperaturas não tão elevadas, determinadas no último trimestre de 2008 (médias das temperaturas mínimas e máximas mensais respectivamente de $13,1^{\circ} \mathrm{C}$ e $22,8^{\circ} \mathrm{C}$ em outubro, $17,2^{\circ} \mathrm{C}$ e $27,0^{\circ} \mathrm{C}$ em novembro, e $17,1^{\circ} \mathrm{C}$ e $27,5^{\circ} \mathrm{C}$ em dezembro).

Nos meses de agosto e setembro, o cultivar Florida Festival produziu maior número de frutos, enquanto a menor produção coube ao 'Sabrosa'. Em agosto, o desempenho do 'Camarosa' e do 'Earlibrite' foi equiparável ao do 'Florida Festival'. Entretanto, em setembro, o número de frutos produzidos pelo cv. Earlibrite não diferiu daquele observado para o 'Sabrosa'. A partir de outubro, o cultivar Camarosa passou a produzir maior número de frutos. Neste mês, distinguiram-se um grupo intermediá- rio, representado pelos cultivares Florida Festival e Sabrosa, e um grupo inferior, com menor produção de frutos, constituído pelo 'Earlibrite'. Em novembro, todos os cultivares encontravam-se no pico da produção, mas continuaram a se distribuir em três grupos distintos, quanto ao número de frutos produzidos: superior ('Sabrosa' e 'Camarosa'), intermediário ('Florida Festival') e inferior ('Earlibrite'). No último período de avaliação (mês de dezembro), o cv. Camarosa manteve-se produzindo elevado número de frutos por planta, maior que o do 'Sabrosa', cujo desempenho foi superior, ainda, ao dos cultivares Earlibrite e Florida Festival (Tabela 3).

$\mathrm{O}$ efeito do período de colheita sobre o número de frutos produzidos pela 'Earlibrite', determinado pelo fotoperíodo e pelas temperaturas ocorridas, foi descrito por modelo linear crescente e, para os cultivares Camarosa,

(a)

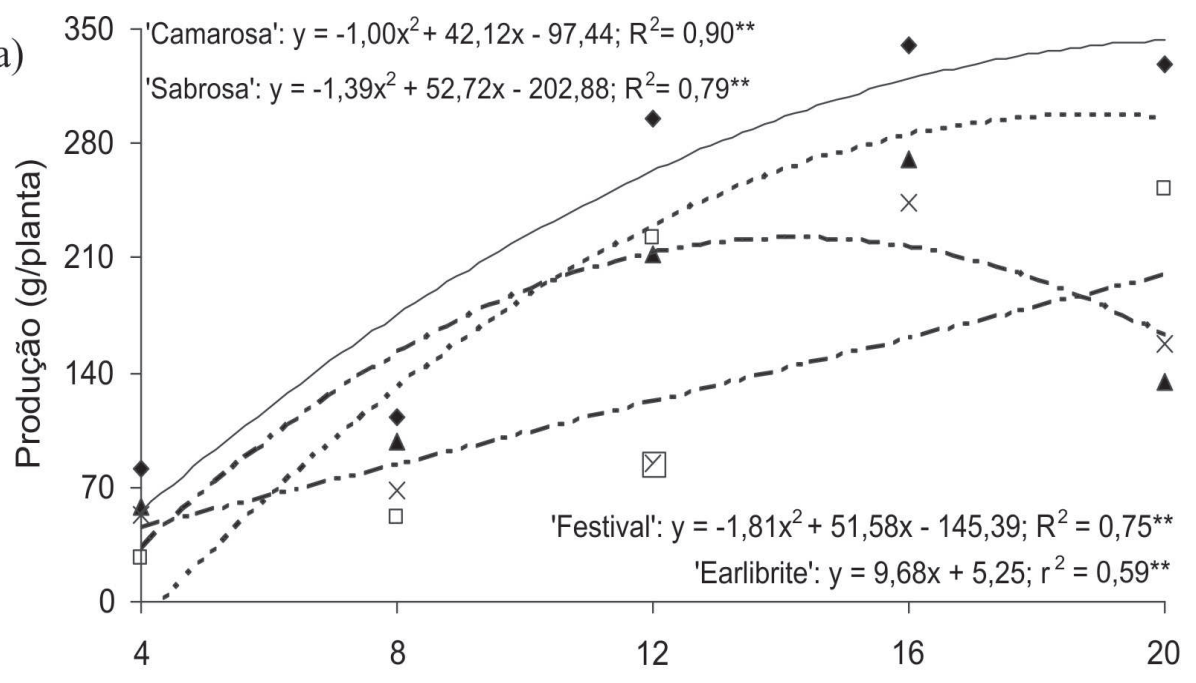

(b)

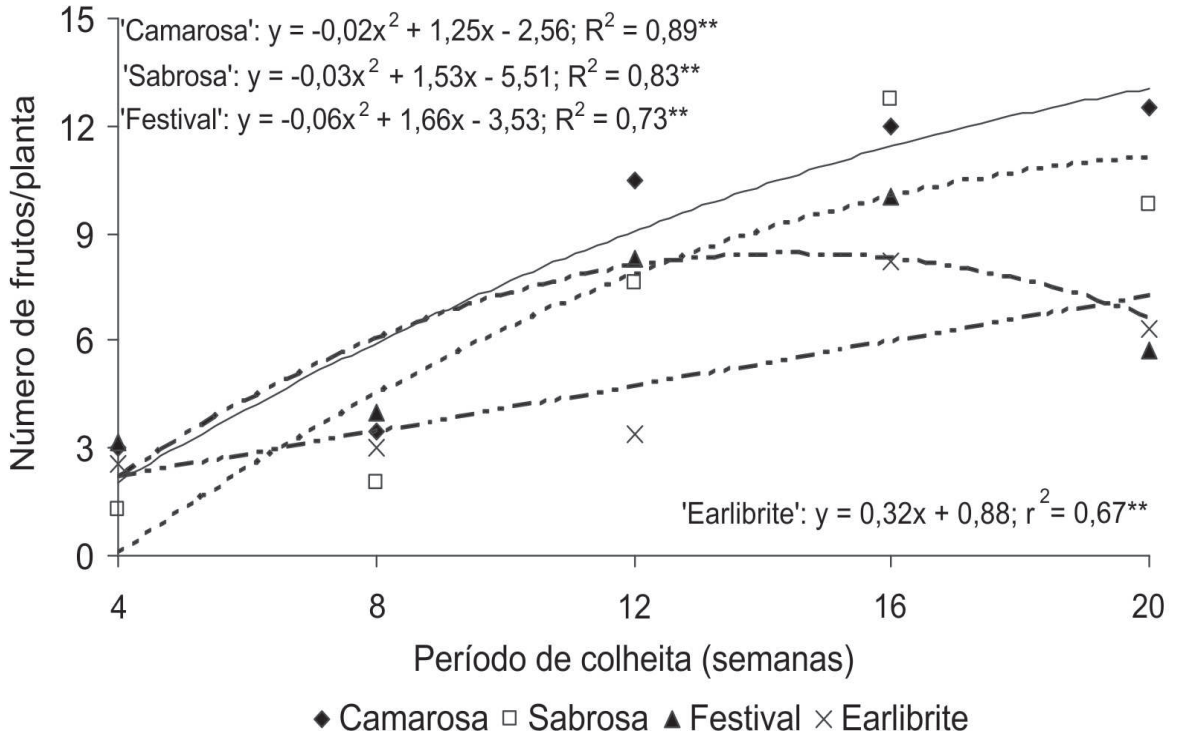

Figura 1. Produção (a) e número de frutos comerciais (b) por planta de quatro cultivares de dia-curto de morangueiro (Fragaria $x$ ananassa Duch.), em função do período de colheita. 
Tabela 3. Número de frutos por planta de quatro cultivares de dia-curto de morangueiro (Fragaria $\mathrm{x}$ ananassa Duch), em função do período de colheita

\begin{tabular}{|c|c|c|c|c|c|}
\hline \multirow{2}{*}{ Cultivar } & \multicolumn{5}{|c|}{ Período de colheita } \\
\hline & Agosto & Setembro & Outubro & Novembro & Dezembro \\
\hline & \multicolumn{5}{|c|}{$\mathrm{N}^{\circ} /$ planta } \\
\hline Camarosa & $3,0 \mathrm{a}$ & $3,5 \mathrm{a}$ & $10,5 \mathrm{a}$ & $12,0 \mathrm{a}$ & $12,5 \mathrm{a}$ \\
\hline Sabrosa & $1,3 \mathrm{~b}$ & $2,0 \mathrm{~b}$ & $7,6 \mathrm{~b}$ & $12,7 \mathrm{a}$ & $9,8 \mathrm{~b}$ \\
\hline Florida Festival & $3,2 \mathrm{a}$ & $4,0 \mathrm{a}$ & $8,3 \mathrm{~b}$ & $10,0 \mathrm{~b}$ & $5,7 \mathrm{c}$ \\
\hline Earlibrite & $2,6 \mathrm{a}$ & $3,0 \mathrm{ab}$ & $3,4 \mathrm{c}$ & $8,0 \mathrm{c}$ & $6,3 \mathrm{c}$ \\
\hline
\end{tabular}

Médias seguidas de mesma letra, nas colunas, não diferem significativamente entre si pelo teste de Tukey a $5 \%$.

Sabrosa e Florida Festival, por modelos quadráticos, com valores máximos estimados correspondentes a 31,2; 25,5; e 13,8 semanas, respectivamente (Figura 1b). Estes resultados mostram que, a exceção do 'Florida Festival', todos os demais não atingiram sua produção máxima de frutos, dentro do período de avaliação. Ressalta-se, porém, que a partir do final de dezembro, a produção de morangueiro na região sul do Rio Grande do Sul é bastante prejudicada, sob o aspecto fitossanitário, em razão da ocorrência de temperaturas elevadas, associadas à umidade relativa do ar também alta, que predispõem à ocorrência de doenças.

Não foi determinado efeito da interação entre os fatores cultivar e período de colheita, sobre a variável massa da matéria fresca média de frutos. Entretanto, destaca-se, que para todos os cultivares avaliados, os valores determinados foram bastante elevados (Tabela 1); no caso do 'Florida Festival' superando, inclusive, os valores médios preconizados por seus detentores $(<20 \mathrm{~g})$ (Chandler et al., 2000b).

Diante dos resultados obtidos, os cultivares Sabrosa, Florida Festival e Earlibrite consistem em novas alternativas varietais para os produtores de morango do sul do Brasil.

\section{CONCLUSÕES}

Os cultivares de morango Earlibrite, Florida Festival e Sabrosa apresentaram alto potencial produtivo, sob condições de produção em túnel baixo, consistindo em novas alternativas varietais para os agricultores de Pelotas-RS.

O 'Sabrosa' proporcionou maior produção acumulada de frutos por planta e maior massa da matéria fresca média que o 'Florida Festival' e o 'Earlibrite'.

Quanto ao número de frutos por planta, 'Sabrosa' e 'Florida Festival' foram mais produtivas que 'Earlibrite'.

\section{AGRADECIMENTOS}

À Fundação de Amparo à Pesquisa do Estado do Rio Grande do Sul - FAPERGS e ao Conselho Nacional de Desenvolvimento Científico e Tecnológico - CNPq, pelo apoio financeiro e pela concessão de bolsa de estudo.

\section{REFERENCIAS}

Antunes LEC \& Reisser Júnior C (2007) Fragole, i produttori brasiliani mirano all' esportazione in Europa. Rivista di Frutticoltura e di Hortofloricoltura, 69:60-64.

Chandler CK \& Sumler Jr. JC (2002) Performance of UF strawberry cultivars planted on three dates in October. Berry Times, 2:1-4.

Chandler CK, Legard DE, Duningan DD, Crocker TE \& Sims CA (2000a) 'Earlibrite' strawberry. HortScience, 35:1363-1365.

Chandler CK, Legard DE, Duningan DD, Crocker TE \& Sims CA (2000b) 'Strawberry Festival' strawberry. HortScience, 35: 13661367.

Estação agroclimatológica de Pelotas (2011) Boletim agroclimatológico. Disponível em: <http://www.cpact.embrapa.br/agromet/ estacao/boletim.html>. Acessado em: 01 de setembro de 2011.

Inotalis - Innovación en variedades vegetales (2010) Candonga. Disponível em: <http://inotalis.com/ index.php?option $=$ com_content\&task=view $\&$ id $=26 \&$ Itemid $=57>$. Acessado em: 07 de julho de 2011.

Leis M, Castagnoli G \& Martinelli A (2002) Naiad Civl35. Rivista di Frutticoltura e di Hortofloricoltura, 64:48-49.

Oliveira RP, Nino AFP \& Scivittaro WB (2005) Mudas certificadas de morangueiro: maior produção e melhor qualidade da fruta. A Lavoura, 108:35-38.

Oliveira RP \& Scivittaro WB (2008) Produção de morangueiro cv. Cegnidarem sob túnel plástico. Ciência Rural, 38:2613-2617.

Oliveira RP, Scivittaro WB \& Finkenauer D (2008) Produção de morangueiro da cv. Camino Real em sistema de túnel. Revista Brasileira de Fruticultura, 30:681-684.

Pagot E \& Hoffmann A (2003) Produção de pequenas frutas. Bento Gonçalves, Embrapa Uva e Vinho. p.9-17. (Embrapa Uva e Vinho. Documentos, 37).

Planasa (2011) Candonga. Disponível em: <http://www.planasa.es/ product/Fresa/candong/candon.htm $>$. Acessado em: 07 de julho de 2011.

Ronque VER (1998) Cultura do morangueiro; revisão e prática. Curitiba, Emater. 206p.

Santos AM (2003) Cultivares. In: Santos AM \& Medeiros ARM (Eds.) Morango: produção. Brasília, Embrapa Informação Tecnológica. p.24-30. (Embrapa Informação Tecnológica. Frutas do Brasil, 40).

Santos HG, Jacomine PKT, Anjos LHC., Oliveira VA, Oliveira JB, Coelho MR, Lumbreras JF \& Cunha TJF (2006) Sistema brasileiro de classificação de solos. $2^{\text {a }}$ ed. Rio de Janeiro, Embrapa Solos. 306p.

Shasta Nursery (2011) Variety list. Disponível em: <http:// www.rootstock.com/variety.html . Acessado em: 07 de julho de 2011. 
Singh A, Patel RK, De LC \& Pereira LS (2008) Performance of strawberry (Fragaria $\mathrm{x}$ ananassa) cultivars under sub-tropics of Meghalaya. Indian Journal of Agricultural Sciences, 78:575580

Sociedade Brasileira de Ciência do Solo. Comissão de Química e Fertilidade do Solo (SBCS-CQFS) (2004) Manual de adubação e calagem para os Estados do Rio Grande do Sul e de Santa Catarina. $10^{\mathrm{a}}$ ed. Porto Alegre, SBCS-CQFS. 400p. 